\title{
Experimental Verification of New Function Theories of Particles (1)- Experimental Verification of New Kinetic Energy Theorem of Rigid Body Fixed Axis Rotation
}

\author{
Yong YANGa, Wei-Ping ZHAO, Xiao-Gang GUO and Zheng-Qiang JING \\ Tongchuan Vocational Technology College, Shaanxi Radio and Television University, Tongchuan, Shaanxi, China
}

\begin{abstract}
Sliding friction torque does dissipative work on the force-bearer, transforming the kinetic energy of the bearer into internal energy and others of the bearer. Sliding friction countertorque does dissipative work on the applying body, transforming the kinetic energy of the bearer into internal energy and others of the applying body. In the experiment, the dissipative work done by sliding friction torque on the bearer is measured and calculated, the increase value of the internal energy of the bearer is measured and calculated, and the increase value of the internal energy of the applying body is measured and calculated. The consistency between their respective dissipative work and their respective internal energy increase value is compared.
\end{abstract}

\section{Introduction}

Conservative force (torque) or non-conservative force (torque) can do non-dissipative work to transfer mechanical energy, and non-conservative force (torque) can also do dissipative work to transform mechanical energy into other forms of energy. Non-conservative force (torque) does dissipative work, and nonconservative reacting force (torque) does the equivalent dissipative work also at the same time. This is a law of motion energy transformation of matter. This natural law is perceptually recognized [1], theoretically proved [2], explored and concluded [3]. Based on energy transformation and conservation laws, Newton's laws of motion, work characteristics of conservative force (torque) and non-conservative force (torque), a new kinetic energy theorem of particle, a new kinetic energy theorem of particle system, a new function principle of particle system and a new kinetic energy theorem of rigid body fixed axis rotation are derived. These 4 new theorems and principles constitute new function theory [4].

\section{Prediction of the new kinetic energy theorem of rigid body fixed axis rotation}

The new kinetic energy theorem of rigid body fixed axis rotation is a reflection of the new function theory, expressed as follows.

In differential expression:

$$
\mathrm{d} A_{\mathrm{cm}}+\mathrm{d} A_{\text {ncmi }}+\mathrm{d} A_{\text {ncmd }}+\mathrm{d} A_{\text {ncrmd }}=\mathrm{d}\left(\frac{1}{2} J \omega^{2}\right)
$$

In integral expression:

$$
A_{\mathrm{cm}}+A_{\mathrm{ncmi}}+A_{\mathrm{ncmd}}+A_{\mathrm{ncrmd}}=\frac{1}{2} J \omega^{2}-\frac{1}{2} J \omega_{0}^{2}
$$

The meaning is that during a rigid body fixed axis rotation process there are two parts of non-dissipative work: $A_{\mathrm{cm}}$ (infinitesimal), work done by all conservative external acting torque on the rigid force-bearer, and $A_{\text {ncmi }}$ (infinitesimal), implicated work done by all nonconservative acting torque on the rigid force-bearer. There are two parts of dissipative work: $A_{\text {ncmd }}$ (infinitesimal), dissipative work done by all nonconservative acting torque on the rigid force-bearer, and $A_{\text {ncmd }}$ (infinitesimal), dissipative work done by all nonconservative reacting torque on the rigid bearer. The sum of these 4 parts of work is equal to the increment (infinitesimal) of the kinetic energy of the rigid body fixed axis rotation.

From the expressions of the new kinetic energy theorem of rigid body fixed axis rotation, we can see that the rotational kinetic energy increment of fixed axis rotation rigid body is determined by the sum of 4 terms of non-dissipative work and dissipative work. So, let the earth's surface be inertial system $\mathrm{S}$, a rigid body for a research object be No. 0, rigid body No.0 with rotary inertia $J_{0}$ to rotating axis $o z$ rotate around the fixed axis $o z$, and the direction of angular speed $\omega$ of the fixed axis rotation rigid body be the reference positive direction of the fixed axis $o z$. Rigid body No.0 in fixed axis rotation is under the effect of $m$ applying rigid bodies. These $m$ applying rigid bodies are in turn No. $j(j=1,2, \cdots, m)$, their rotary inertias to fixed axis $o z$ are in turn $J_{\mathrm{j}}$.

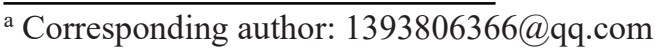


Scene 1: The resultant conservative torque $\mathrm{M}_{(\mathrm{cm}) 0}$ does non-dissipative work on the fixed axis rotation rigid body. Implicated work $\mathrm{d} A_{(\mathrm{ncmi})}=\mathrm{M}_{(\mathrm{ncm}) 0 \mathrm{j}} \cdot \mathrm{d} \theta_{\mathrm{j}}$ done by static friction torque $\mathrm{M}_{(\mathrm{ncm}) 0 j}$ on the fixed axis rotation rigid body is also dissipative work and can change kinetic energy of the rigid force-bearer fixed axis rotation. This is a law described by the classical kinetic energy theorem of rigid body fixed axis rotation and has been verified [5].

Scene 2: If the fixed axis rotation rigid body is only under the effect of the non-conservative torque $\mathrm{M}_{(\mathrm{ncm}) 0 \mathrm{j}}$, non-conservative torque $\mathrm{M}_{(\mathrm{ncm}) 0 \mathrm{j}}$ would generally do both implicated work $A_{\text {ncmi }}$ and dissipative work $A_{\text {ncmd }}$ on the rigid force-bearer $J_{0}$. Non-conservative countertorque $\mathrm{M}_{\text {(ncm)j0 }}$ does dissipative work $A_{(\mathrm{ncrmd)j}}$ on the applying rigid body $J_{\mathrm{j}}$ also at the same time. The dissipative work done by non-conservative torque and non-conservative countertorque is related to both the non-conservative torque on the rigid force-bearer and the relative angular displacement $\mathrm{d} \theta_{0 \mathrm{j}}$ and $\mathrm{d} \theta_{\mathrm{j} 0}$ of the force-bearing point relative to the applying point.

That is, under the condition of

$$
\left\{\begin{array}{l}
\mathbf{M}_{(\mathrm{cm}) 0}=\sum_{\mathrm{j}=1}^{\mathrm{m}} \mathbf{M}_{(\mathrm{cm}) \mathrm{j}} \equiv 0 \\
\mathrm{~d} A_{\mathrm{cm}}=\mathbf{M}_{(\mathrm{cm}) 0} \cdot \mathrm{d} \boldsymbol{\theta}_{0} \equiv 0
\end{array}\right.
$$

Form (1) and (4), we can obtain

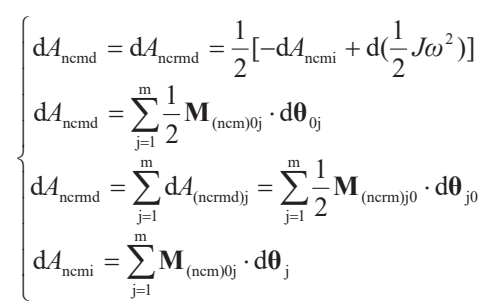

From (5), we can see that the sliding friction torque does dissipative work and the sliding friction countertorque does equivalent dissipative work at the same time. Both transform the kinetic energy of rigid body $J_{0}$ into non-mechanical energy of respective forcebearers. If wear is neglected, rigid body $J_{0}$ for the research object will be regarded as a thermodynamic system. When the thermodynamic system in an adiabatic process $\left(\mathrm{d} Q_{0}=0\right)$, we can, based on the first law of thermodynamics $\mathrm{d} Q_{0}=\mathrm{d} U+\mathrm{d} A$, obtain

$$
\left\{\begin{array}{l}
\mathrm{d} Q=\mathrm{d} U+\mathrm{d} A, \mathrm{~d} Q_{0}=0 \\
\mathrm{~d} U_{0}=-\mathrm{d} A_{\text {nemd }}=-\sum_{\mathrm{j}=1}^{\mathrm{m}} \frac{1}{2} \mathbf{M}_{(\mathrm{ncm}) \mathrm{j}_{\mathrm{j}}} \cdot \mathrm{d} \boldsymbol{\theta}_{0 \mathrm{j}} \\
\mathrm{d} U_{0}=-\mathrm{d} A_{\text {nemd }}=-\mathrm{d} A_{\text {nemd }}=\sum_{\mathrm{j}=1}^{\mathrm{m}} \mathrm{d} U_{\mathrm{j}}
\end{array}\right.
$$

That is, in an adiabatic process, the sliding friction torque does dissipative work and the sliding friction countertorque does equivalent dissipative work at the same time. Both transform the kinetic energy of rigid body $J_{0}$ into internal energy increment infinitesimals $\mathrm{d} U_{0}$ and $\mathrm{d} U_{\mathrm{j}}$ of respective force-bearers. From (10) and (11), we can obtain

$$
\mathrm{d} U_{0}=\sum_{\mathrm{j}=1}^{\mathrm{m}} \mathrm{d} U_{\mathrm{j}}=-\sum_{\mathrm{j}=1}^{\mathrm{m}} \frac{1}{2} \mathbf{M}_{(\mathrm{ncm}) 0 \mathrm{j}} \cdot \mathrm{d} \boldsymbol{\theta}_{0 \mathrm{j}}
$$

In the adiabatic process with wear neglected, dissipative work done by all sliding friction torque on rigid body $J_{0}$ transformed into total infinitesimal $\mathrm{d} U_{0}$ of internal energy increment of rigid body $J_{0}$, is equal to dissipative work done by all sliding friction countertorque on applying rigid body $J_{\mathrm{j}}$ transformed into internal energy increment infinitesimal $\sum_{\mathrm{j}=1}^{\mathrm{m}} \mathrm{d} U_{\mathrm{j}}$ of rigid body $J_{\mathrm{j}}$.

This is the prediction of the new kinetic energy theorem of rigid body fixed axis rotation on numerical relation that in the adiabatic process with wear neglected, all sliding friction torque and all sliding friction countertorque do dissipative work on their respective force-bearers and transform the kinetic energy of rigid body $J_{0}$ into internal energy increment infinitesimal of their respective force-bearers. This prediction needs experimental verification.

\section{The experiment devices and principle}

\subsection{The experiment devices}

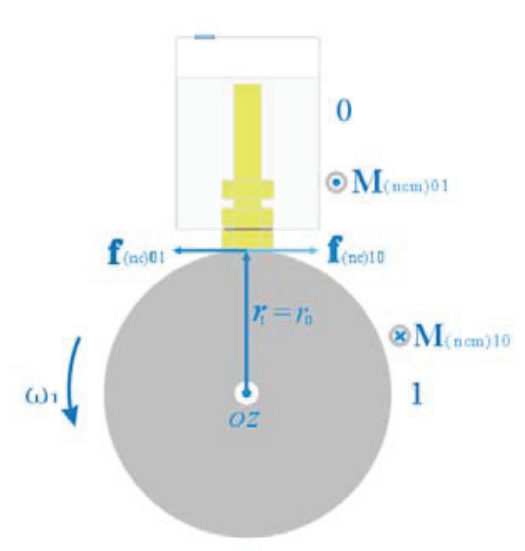

Figure 1. Two friction pairs

As shown in Figure 1, a cylindrical iron flywheel as the rotating friction pair is mounted at the left end of a singlephase motor shaft; another fixed combined friction pair composed of brass bolt, transparent hard plastic bottle and water is supported just above the iron flywheel cylinder. When the motor drives the iron flywheel to rotate synchronously, sliding friction occurs between flywheel cylindrical surface and the lower surface of the brass bolt of the fixed combined friction pair. A reduction gear box is installed at the right end of the motor shaft. The output end of the reduction gear box is connected with a rotary counter used to record revolution of rotary friction pair flywheel. Dynamometer, electronic scales, alcohol thermometer, calorimeter, infrared thermometer are necessary. 


\subsection{The verification experiment principle}

In the process of rigid body fixed axis rotation, the direction of sliding friction torque vector $\mathrm{M}_{(\mathrm{ncm}) 0 \mathrm{j}}$ is opposite to the direction of relative angular displacement vector $\mathrm{d} \theta_{0 \mathrm{j}}$ of the force-bearing point relative to the applying point. From (10) and (11), we obtain

$$
\begin{aligned}
& \mathrm{d} A_{\text {nemd }}=\sum_{\mathrm{j}=1}^{\mathrm{m}} \frac{1}{2} \mathbf{M}_{(\mathrm{ncm}) \mathrm{j}} \cdot \mathrm{d} \boldsymbol{\theta}_{0 \mathrm{j}} \\
& =\sum_{\mathrm{j}=1}^{\mathrm{m}} \frac{1}{2}\left(M_{(\mathrm{ncm}) \mathrm{j}_{\mathrm{j}}} \mathbf{k}\right) \cdot\left(-\mathbf{k d} \theta_{0 \mathrm{j}}\right) \\
& =\sum_{\mathrm{j}=1}^{\mathrm{m}}\left(-\frac{1}{2}\right) M_{(\mathrm{ncm}) \mathrm{j}) \mathrm{j}} \mathrm{d} \theta_{0 \mathrm{j}} \\
& =\mathrm{d} A_{\text {nermd }}
\end{aligned}
$$

Substituting (13) into (12) obtains

$$
\begin{aligned}
\mathrm{d} U_{0} & =-\sum_{\mathrm{j}=1}^{\mathrm{m}}-\left(\frac{1}{2} M_{(\mathrm{ncm}))_{\mathrm{j}} \mathrm{j}} \mathrm{d} \theta_{0 \mathrm{j}}\right) \\
& =\sum_{\mathrm{j}=1}^{\mathrm{m}} \mathrm{d} U_{\mathrm{j}}
\end{aligned}
$$

That is, for single direction fixed axis rotation rigid body in the process of sliding friction without wear, internal energy increment infinitesimal is equal to the negative value of total infinitesimal of dissipative work done by sliding friction torque, or equal to internal energy increment infinitesimal of applying rigid bodies transformed from dissipative work done by all sliding friction countertorque on all applying rigid bodies.

In integral expression is

$$
\begin{aligned}
\Delta U_{0} & =-\sum_{j=1}^{m}-\left(\frac{1}{2} M_{(\text {(nem) }) j} \Delta \theta_{0 j}\right) \\
& =\sum_{j=1}^{m} \Delta U_{j}
\end{aligned}
$$

In the experiment, the internal energy increment of rigid bodies $J_{0}$ and $J_{\mathrm{j}}$ can be measured and calculated on the basis of the following formulas.

$$
\begin{aligned}
& \Delta U_{0}=m_{0} c_{0}\left(T_{01}-T_{00}\right) \\
& \Delta U_{\mathrm{j}}=m_{\mathrm{j}} c_{\mathrm{j}}\left(T_{\mathrm{j} 1}-T_{\mathrm{j} 0}\right)
\end{aligned}
$$

The dissipative work done by the sliding friction torque on rigid body $J_{0}$ can be measured and calculated on the basis of the following formulas.

$$
\begin{gathered}
-A_{\mathrm{ncmd}}=\sum_{\mathrm{j}=1}^{\mathrm{m}} \frac{1}{2} M_{(\mathrm{ncm}) 0 \mathrm{j}} \Delta \theta_{0 \mathrm{j}}=-\mathrm{d} A_{\mathrm{ncrmd}} \\
M_{(\mathrm{ncm}) 0 \mathrm{j}}=f_{0 \mathrm{j}} r_{0} \\
\Delta \theta_{0 \mathrm{j}}=2 \pi\left|n_{0 \mathrm{j}}\right|
\end{gathered}
$$

In the experiment, when the cylindrical flywheel as the rotary friction pair starts rotating around a fixed axis with a stationary state, sliding friction occurs once the fixed friction pair into contact with the flywheel cylindrical surface, and the flywheel stops rotating after number of turns. Based on the new kinetic energy theorem of rigid body fixed axis rotation, dissipative work done by sliding friction torque and sliding friction countertorque on their respective force-bearing friction pairs can be calculated, and internal energy increment of two friction pairs during friction can be measured.

As the research object, the number of the fixed friction pair is 0 . The number of the rotating friction pair flywheel, as the applying rigid body of friction torque, is 1 . The number of the earth is 3 .

In the whole process of rigid body No.0 under friction, only the sliding friction torque $\mathrm{M}_{(\mathrm{ncm}) 01}$ does dissipative work $A_{\text {ncmd }}$ and implicated work $A_{\text {ncmi }}$ on rigid body No.0. No other torque does work on rigid body No. 0 .

From (18), (19) and (20), we obtain

$$
\begin{aligned}
-A_{\text {ncmd }} & =\sum_{\mathrm{j}=1}^{1} \frac{1}{2} M_{(\mathrm{ncm}) 0 \mathrm{j}} \Delta \theta_{0 \mathrm{j}} \\
& =\frac{1}{2} M_{(\mathrm{ncm}) 01} \Delta \theta_{01} \\
& =\frac{1}{2} f_{01} r_{0}\left(2 \pi\left|n_{01}\right|\right) \\
& =-A_{\text {ncrmd }} \\
& =\Delta U_{0} \\
& =\Delta U_{1}
\end{aligned}
$$

(21) Is a calculation expression of dissipative work done by sliding friction torque and sliding friction countertorque on their respective force-bearing friction pairs, or a calculation expression of the theoretical value of internal energy of force-bearing friction pairs transformed from mechanical energy of rigid body No.0 as the research object with deformation energy consumption neglected.

$$
\begin{aligned}
A_{\mathrm{ncmi}} & =\sum_{\mathrm{j}=1}^{1} M_{(\mathrm{ncm}) 0_{\mathrm{j}}} \Delta \theta_{\mathrm{j}} \\
& =M_{(\mathrm{ncm}) 01} \Delta \theta_{1} \\
& =2 \pi n_{1} f_{01} r_{0}
\end{aligned}
$$

(22) Is a calculation expression that sliding friction torque does implicated work $A_{\text {ncmi }}$ on rigid body No.0, and transfers mechanical energy of applying rigid body No.1 into mechanical energy of rigid body No.0, as the source of mechanical energy of rigid body No.0.

\section{Experiment procedure}

a. Mass $m_{0}$ and specific heat $c_{0}$ of combined friction pair No. 0 and the positive pressure $\mathrm{N}_{01}$ on friction surface are measured and recorded. Sliding friction coefficient $\mu_{01}$ is checked and recorded. Sliding friction $\mathrm{f}_{01}$ is calculated. Mass $m_{1}$ and radius $r_{1}$ of friction pair No.1 are measured and recorded. Specific heat $c_{1}$ is checked and recorded. Ambient temperature $298 \mathrm{k}$.

b. According to Fig.1, the experimental devices are assembled with $220 \mathrm{~V}$ three-hole power socket.

c. Initial temperature $T_{00}$ of combined friction pair No. 0 is measured with the alcohol thermometer and recorded. Initial temperature $T_{10}$ of friction pair No.1 is measured with calorimeter. Rotary counter is reset.

d. The power plug of single-phase motor is into the power socket. The motor is started. 
Table 1. Non-mechanical energy and internal energy increment transformed to friction pair No.0 (SI system)

\begin{tabular}{|c|c|c|c|c|c|c|c|c|c|c|c|}
\hline No. & $f_{\text {(nc) } 01}$ & $r_{0}=r_{1}$ & $M_{\text {(ncm) } 01}$ & $\left|n_{01}\right|=n_{1}$ & $-A_{\text {ncmd }}$ & $m_{0}$ & $c_{0}$ & $T_{00-273.1}$ & $T_{01-273.1}$ & $\Delta U_{0}$ & $E_{\mathrm{r}}$ \\
\hline 1 & 2 & 0.0375 & 0.075 & 2000 & 471.00 & 0.138 & 2038.1 & 25.5 & 27.1 & 450.01 & 0.0446 \\
\hline 2 & 2 & 0.0375 & 0.075 & 2100 & 494.55 & 0.138 & 2038.1 & 24.8 & 26.5 & 478.14 & 0.0332 \\
\hline 3 & 2 & 0.0375 & 0.075 & 2200 & 518.10 & 0.138 & 2038.1 & 24.7 & 26.5 & 506.26 & 0.0228 \\
\hline
\end{tabular}

Table 2. Non-mechanical energy and internal energy increment transformed to friction pair No.1 (SI system)

\begin{tabular}{|c|c|c|c|c|c|c|c|}
\hline No. & $-A_{\text {ncrnd }}=-A_{\text {ncond }}$ & $m_{1}$ & $c_{1}$ & $T_{10}-273.1$ & $T_{11}-273.1$ & $\Delta U_{1}$ & $E_{\mathrm{r}}^{\prime}$ \\
\hline 1 & 471.00 & 0.602 & 444 & 25.8 & 27.5 & 454.390 & 0.0353 \\
\hline 2 & 494.55 & 0.602 & 444 & 24.8 & 26.6 & 481.118 & 0.0272 \\
\hline 3 & 518.10 & 0.602 & 444 & 25.1 & 27.0 & 507.847 & 0.0198 \\
\hline
\end{tabular}

e. Attention to the running condition of friction pair is taken. The speed control knob can be used to adjust rated output power of the motor.

f. When the rotary counter is up to 2000 revolutions, the motor is stopped.

g. A thermal insulation pad is put at the contact surface of two friction pairs, and temperature $T_{01}$ of combined friction pair No.0 is measured and recorded. The final average temperature $T_{11}$ of friction pair No.1 is measured, calculated and recorded.

h. The experimental data are handled.

The relative errors in the tables

$$
\begin{gathered}
E_{\mathrm{r}}=\frac{\left|A_{\text {ncmd }}\right|-\Delta U_{0}}{\left|A_{\text {ncmd }}\right|} \\
E_{\mathrm{r}}^{\prime}=\frac{\left|A_{\text {ncrmd }}\right|-\Delta U_{1}}{\left|A_{\text {ncrmd }}\right|}
\end{gathered}
$$

\section{Experimental matters needing attention}

It is the value of dissipative work done by sliding friction torque on the research object, the value of internal energy increment of the research object and the equivalent relation between the two values that are measured in this verification experiment. Unlike traditional, only the value of work done by sliding friction torque on a single forcebearer is calculated, and the total value of internal energy increment of two parties in mutual friction is measured[6], as well as the equivalent relation between the two values. The work done by sliding friction countertorque on the single applying body is placed to the sliding friction torque.

For the contact sliding friction between the rigid bodies, thermal transmission between friction bodies has great influence on the experimental results [7]. Therefore, material making two friction pairs with approximately equal heat capacity, better heat transfer and similar thermal conductivity is selected. So, two friction pairs with the same initial temperature can obtain the same internal energy increment in the sliding friction process, and temperature difference always close to zero has little influence on heat transfer between them. The energy transformed from sliding friction is kept in their friction bodies as far as possible.

At the same time, there is heat transfer between two friction pairs and the environment matters also. In order to reduce experimental errors, the difference between the final temperature of friction pair and the ambient temperature in each experiment is not too much. The temperature difference in this experiment was within the range of $3 \mathrm{~K}$.

\section{Conclusions}

From Table 1 and Table 2, we can see, the value of dissipative work done by a pair of sliding friction torque on their respective force-bearing friction pairs is equal to the value of internal energy increment of the friction pairs in the error range. The new kinetic energy theorem of rigid body fixed axis rotation can reflect a law of the nature.

\section{References}

1. Y. Yang, Journal of Shaanxi Normal University (Natural Science Edition),30, 24-26 (2002)

2. Y. Yang, Journal of Shaanxi Normal University (Natural Science Edition),33,12-15,292 (2005)

3. Y. Yang, Manufacturing Automation,12,126-128 (2009)

4. Y. Yang, Proceedings of the 2015 International Conference on Advanced Material Engineering, 65$83(2015)$

5. X. C. Ren, Y. Q. Wang, N. G. Yang, Journal of Yan'an University (Natural Sciences), 20 (1),4950(2001)

6. Y. L. Ma, Journal of Yanbei Teachers College,12(3),70-71(1996)

7. C.G. Cui , P. Y. Tian, Y. Tian, Lubrication Engineering,42(3):23-27,10(2017) 